Acta Crystallographica Section F

Structural Biology

and Crystallization

Communications

ISSN 1744-3091

\section{Pereira, ${ }^{a}$ I. H. Saraiva, ${ }^{a}$ \\ R. Coelho, ${ }^{a}$ D. K. Newman, ${ }^{b}$ \\ R. O. Louro ${ }^{a}$ and C. Frazão ${ }^{a *}$}

anstituto de Tecnologia Química e Biológica, Universidade Nova de Lisboa, Apartado 127, 2781-901 Oeiras, Portugal, and ${ }^{\mathbf{b}}$ California Institute of Technology/Howard Hughes Medical Institute, 1200 East California Boulevard, Mail Code 147-75, Pasadena, CA 91125, USA

Correspondence e-mail: frazao@itqb.unl.pt

Received 22 June 2012

Accepted 18 July 2012

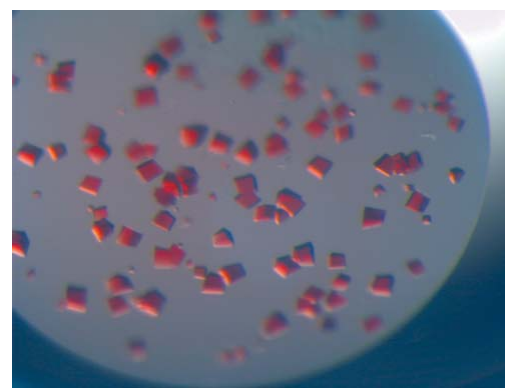

(C) 2012 International Union of Crystallography All rights reserved

\section{Crystallization and preliminary crystallographic studies of FoxE from Rhodobacter ferrooxidans $\mathrm{SW} 2$, an Fe" oxidoreductase involved in photoferrotrophy}

FoxE is a protein encoded by the foxEYZ operon of Rhodobacter ferrooxidans $\mathrm{SW} 2$ that is involved in $\mathrm{Fe}^{\mathrm{II}}$-based anoxygenic photosynthesis ('photoferrotrophy'). It is thought to reside in the periplasm, where it stimulates lightdependent $\mathrm{Fe}^{\mathrm{II}}$ oxidation. It contains 259 residues, including two haem $c$-binding motifs. As no three-dimensional model is available and there is no structure with a similar sequence, crystals of FoxE were produced. They diffracted to $2.44 \AA$ resolution using synchrotron radiation at the Fe edge. The phase problem was solved by SAD using SHELXC/D/E and the experimental maps confirmed the presence of two haems per molecule.

\section{Introduction}

FoxE is a di-haem c-type cytochrome encoded by the foxEYZ operon from the bacterium Rhodobacter ferrooxidans SW2, a bacterium that is capable of photoferrotrophy. This is a metabolic strategy that is characterized by the utilization of ferrous iron $\left(\mathrm{Fe}^{\mathrm{II}}\right)$ oxidation as the sole source of electrons for photosynthesis (Widdel et al., 1993). The ferric iron $\left(\mathrm{Fe}^{\mathrm{III}}\right)$ resulting from this metabolism precipitates as ferric (hydr)oxides at the neutral $\mathrm{pH}$ at which this organism grows. This ancient form of photosynthesis (Xiong et al., 2000) is hypothesized to have catalyzed the deposition of ancient sedimentary deposits known as banded iron formations in early phases of the history of the Earth (Kappler et al., 2005).

Heterologous expression of the fox operon in the bacterium R. capsulatus SB1003 resulted in enhanced photosynthetic $\mathrm{Fe}^{\mathrm{II}}$ oxidation activity, which suggested that the products of this operon are required for photoferrotrophy (Croal et al., 2007). That the expression of fox $E$ alone is enough to enhance photosynthetic $\mathrm{Fe}^{\mathrm{II}}$ oxidation indicates that this cytochrome is responsible for the $\mathrm{Fe}^{\mathrm{II}}$-oxidation step in this metabolism. Previous functional characterization of FoxE (Saraiva et al., 2012) demonstrated that it is thermodynamically and kinetically capable of oxidizing $\mathrm{Fe}^{\mathrm{II}}$ in vitro.

In-depth characterization of the proteins involved in photoferrotrophy is required in order to understand this ancient form of photosynthesis which is likely to have had a profound impact on the geochemical evolution of the Earth in the past.

\section{Materials and methods}

\subsection{Protein expression and purification}

FoxE was cloned, expressed and purified as described by Saraiva et al. (2012). The protein purity was checked by SDS-PAGE and the pure protein was concentrated using Vivaspin concentrators with a $30 \mathrm{kDa}$ cutoff membrane, leading to a final protein solution consisting of $15-18 \mathrm{mg} \mathrm{ml}^{-1}$ FoxE in $5 \mathrm{~m} M$ potassium phosphate buffer $\mathrm{pH}$ 7.0.

\subsection{Protein crystallization}

Initial FoxE crystallization screens were performed at room temperature $(293 \mathrm{~K})$ by the vapour-diffusion method with commercially available solution kits in a Mini-Bee MicroSys 4000XL Cartesian Dispensing Systems robot (Genomic Solutions, USA). Drops were produced by dispensing $100 \mathrm{nl}$ protein solution plus $100 \mathrm{nl}$ precipitant solution and were equilibrated against $100 \mu \mathrm{l}$ 
precipitant solution; they were examined under a magnifying glass to detect possible crystallization hints. These were then further optimized in microlitre-scale drops by variation of the initial conditions, by the use of crystallization additives and by attempting seeding experiments.

Two crystallization screens, Structure Screen 1 and Structure Screen 2 from Molecular Dimensions Ltd (Suffolk, England), were initially tried. No crystallization hits were detected among those $2 \times$ 48 trials, but precipitation was observed in $\sim 50 \%$ of the drops with solutions containing PEG and in $\sim 60 \%$ of the drops with $\mathrm{pH}$ below 6.5. MacroSol, JCSG-plus and Stura Footprints screens from Molecular Dimensions were then tried. Three distinct crystal shapes were obtained from these 192 conditions: needle-like crystals were obtained using solution 2.16 of MacroSol [0.1 $M$ HEPES $\mathrm{pH} 7.5$, $2.0 \mathrm{M}$ ammonium sulfate, $5 \%(v / v)$ PEG 400], polyhedral crystals with similar shapes were obtained using four solutions from JCSG-plus that contained PEG, namely solution 1.2 [0.1 $M$ sodium citrate $\mathrm{pH}$ 5.5, 20\%(w/v) PEG 3K], solution 1.45 [0.17 $M$ ammonium sulfate, $25.5 \%(w / v)$ PEG $4 \mathrm{~K}, 15 \%$ glycerol], solution 1.21 [0.1 $M$ citrate $\mathrm{pH}$, $20 \%(w / v)$ PEG $6 \mathrm{~K}]$ and solution $2.45[0.2 M$ lithium sulfate, $0.1 M$ bis-Tris $\mathrm{pH} 5.5,25 \%(w / v)$ PEG 3350], and agglomerated polycrystals were obtained using Stura Footprints solution B6 (1.32 M sodium/ potassium phosphate $\mathrm{pH} 7$ ). The conditions that delivered the bestlooking crystals were optimized on the microlitre scale, aiming to control nucleation and to increase the crystal size. However, the use of JCSG-plus solution 1.2 could not be reproduced and solution 1.45 only produced crystals at $303 \mathrm{~K}$. The use of PEGs of sizes $2-6 \mathrm{~K}$ at $5-20 \%$, the addition of 5 or $15 \%$ glycerol, the removal of sodium citrate, the use of drops with different volumes of protein and precipitant $(2: 1.5,2: 1,2.2: 0.8$ and 2.3:0.7), the addition of silicone, paraffin or PEG 400 to control vapour diffusion and the use of macroseeding were attempted. These attempts revealed that the crystals were often not reproducible and showed a gel-like consistency within 4-7 d, therefore not being amenable to X-ray diffraction.

We proceeded with the non-PEG lead, Stura Footprints solution B6 based on phosphate precipitant. Although its microlitre scale-up was reproducible at all temperatures tested (277, 293 and $303 \mathrm{~K}$ ), diffraction experiments showed that the crystals were disordered. Crystallization conditions at $293 \mathrm{~K}$ were then varied using PEGs of sizes 2-6K, 10-36\%(v/v) PEG concentration, 0.1-1.4 $M$ phosphate concentration, $\mathrm{pH}$ values in the range 5-7.2 and different (1:2,1:1 and $2: 1$ ) dispensing ratios. Better crystals grew in $0.2 \mathrm{M}$ sodium/potassium phosphate $\mathrm{pH} 5,20 \%(v / v)$ PEG $5 \mathrm{~K}$, but degraded over time and did not produce usable diffraction. In order to sample wider crystal-

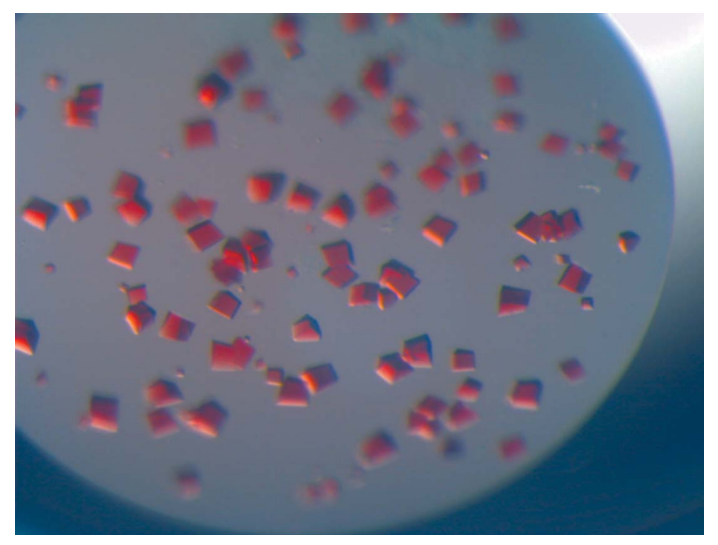

Figure 1

Crystallization drop of FoxE showing red well shaped polyhedral crystals with approximate dimensions of $0.1 \times 0.1 \times 0.02 \mathrm{~mm}$.
Table 1

Crystallographic and diffraction data statistics.

Values in parentheses are for the highest resolution shell.

\begin{tabular}{ll}
\hline ESRF beamline & ID23-1 \\
Wavelength $(\AA)$ & 1.73925 \\
Resolution $(\AA)$ & $57.83-2.44(2.54-2.44)$ \\
Space group & $P 3_{1} 21$ \\
Unit-cell parameters $(\AA)$ & $a=b=112.77, c=143.54$ \\
No. of measured reflections & 1293342 \\
No. of unique reflections & $38526(3591)$ \\
Multiplicity & $32.2(6.3)$ \\
Mean $I / \sigma(I)$ & $28.3(1.9)$ \\
$R_{\text {p.i.m. } \dagger(\%)}(\%)$ & $2.2(25.0)$ \\
$R_{\text {merge }}(\%)$ & $13.6(70.7)$ \\
Completeness $(\%)$ & $96.1(77.1)$ \\
Solvent content $(\%)$ & 52.3 \\
Wilson $B$ factor $\left(\AA^{2}\right)$ & 49.1
\end{tabular}

$\dagger R_{\text {p.i.m. }}=\sum_{h k l}\{1 /[N(h k l)-1]\}^{1 / 2} \sum_{i}\left|I_{i}(h k l)-\langle I(h k l)\rangle\right| / \sum_{h k l} \sum_{i} I_{i}(h k l)$, where $N$ is the data multiplicity, $I_{i}(h k l)$ is the observed intensity and $\langle I(h k l)\rangle$ is the average intensity of multiple observations from symmetry-related reflections. It is an indicator of the precision of the final merged and averaged data set (Weiss, 2001). $\ddagger R_{\text {merge }}=$ $\sum_{h k l} \sum_{i}\left|I_{i}(h k l)-\langle I(h k l)\rangle\right| / \sum_{h k l} \sum_{i} I_{i}(h k l)$, where $I_{i}(h k l)$ is the observed intensity and $\langle I(h k l)\rangle$ is the average intensity of multiple observations from symmetry-related reflections.

lization conditions using phosphate precipitant, the Additive Screen from Hampton Research (Aliso Viejo, USA) was also used, restricted to its 80 nonvolatile conditions. The best crystals were obtained at $293 \mathrm{~K}$ in sitting drops consisting of $1 \mu 115 \mathrm{mg} \mathrm{ml}^{-1}$ protein solution plus $1 \mu \mathrm{l}$ precipitant solution (1.2 $\mathrm{M}$ sodium/potassium phosphate $\mathrm{pH}$ 7, $50 \mathrm{~m} M$ copper chloride) equilibrated against $500 \mu \mathrm{l}$ precipitant solution in the well. Red polyhedral crystals reached dimensions of up to approximately $0.1 \times 0.1 \times 0.02 \mathrm{~mm}$ (Fig. 1). Crystal cryoprotection was accomplished by soaking the crystals in mother liquor complemented with $25 \%(v / v)$ glycerol.

\subsection{X-ray diffraction data collection and crystal phase-problem solution}

The diffraction of a cryostabilized crystal was measured near the iron absorption edge using an ADSC Q315R detector at station ID23-1 of the European Synchrotron Radiation Facility (ESRF), Grenoble, France. Diffraction images from two $360^{\circ}$ crystal rotations, interleaved by a crystal translation in order to start each diffraction run from an unexposed crystal region, were integrated and scaled with $X D S$ (Kabsch, 2010) and the resulting two sets of intensities were merged together with XPREP (Bruker).

The phase problem was solved by single-wavelength anomalous diffraction (SAD) using the HKL2MAP GUI (Pape \& Schneider, 2004; Sheldrick, 2008), which (i) relies on SHELXC to analyse the data, estimate anomalous difference factors and produce initial phase shifts for reflections corresponding to the largest normalized anomalous differences, (ii) uses SHELXD to determined the anomalous heavy-atom substructure and (iii) uses $S H E L X E$ to produce iterative phase improvement by density modification, including automated poly-Ala chain tracing (Sheldrick, 2010).

\section{Results and discussion}

Despite several attempts to optimize the initial FoxE crystallization conditions from PEG- or phosphate-containing solutions, the crystals were not always reproducible; their diffraction indicated disordered crystal packing and showed severe intensity reduction within a few days, when the crystals became gel-like red polyhedra. The addition of copper chloride as an additive to the mother liquor, however, led to increased crystal stability and to increased diffraction capability. 


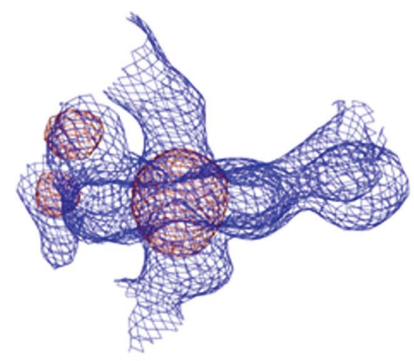

(a)

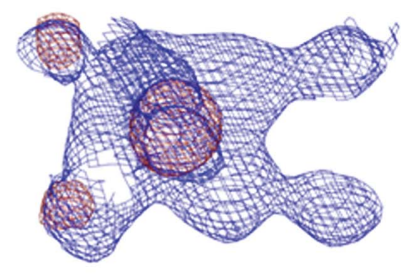

(b)
Figure 2

Experimental SAD electron density (blue mesh, $0.7 \sigma$ ) and anomalous density (red mesh, $5 \sigma$ ) of a representative region of FoxE showing a haem and its ligands. (a) and $(b)$ show views with the haem plane perpendicular and parallel to the paper, respectively. The Fe-atom localization is recognisable in the anomalous map at the haem centre and the thioether $\mathrm{S}$ atoms are recognisable at the haem periphery. The maps were produced with SHELXE (Sheldrick, 2010) and the figures were produced with $P y M O L$ (DeLano, 2002).

FoxE crystallized in space group $P 3_{1} 21$ or $P 3_{2} 21$, with unit-cell parameters $a=b=112.77, c=143.54 \AA$, and the crystals diffracted to $2.44 \AA$ resolution (see Table 1 for further crystallographic details and diffraction data statistics).

The diffraction data measured using X-rays at the Fe absorption edge contained significant anomalous signal up to $3.7 \AA$ resolution, where the self-anomalous correlation coefficient remained above $30 \%$. The probability distribution of the Matthews coefficient for $2.5 \AA$ resolution indicated a 74\% probability of four FoxE molecules in the asymmetric unit, in contrast to a $17 \%$ probability of an asymmetric unit containing only three molecules (Kantardjieff \& Rupp, 2003). As the FoxE sequence contains two haem $c$ cytochrome CXXCH motifs (Croal et al., 2007), SHELXD was set to search for eight $\mathrm{Fe}$ atoms. However, only six anomalous atoms were found with occupancies in the range 0.8-1.0; further hypothetical sites refined to occupancies below 0.2 and were discarded. Accordingly, the FoxE crystal contained three molecules in the asymmetric unit, corresponding to a calculated crystal solvent content of $52 \%$ (Matthews, 1968). SHELXE was run twice. The first run, using phase information from the six-Fe-atom substructure, solved the space-group hand ambiguity, produced initial experimental maps, traced a 641 poly-Ala residue model in 35 chains and listed a set of further anomalous sites. These were examined with Coot (Emsley et al., 2010) to determine the
12 thioether sites that attach the six haems to the three FoxE molecules. A second SHELXE run was thus performed including the phases from a further $12 \mathrm{~S}$ atoms and produced the final experimental maps (Fig. 2), in which 678 poly-Ala residues of the expected 777 were traced in a model with 18 chains and a correlation coefficient of $35 \%$.

Three-dimensional structure determination and refinement of FoxE is in progress in order to unravel the molecular arrangement of this $c$-type cytochrome with unprecedented primary structure that is capable of oxidizing iron in vitro in a $\mathrm{pH}$-dependent manner that appears to be designed to prevent incrustation of the cells by ferric iron.

The authors gratefully acknowledge the ESRF, Grenoble, France for provision of synchrotron radiation and thank Susana Gonçalves, ITQB-UNL, Portugal for diffraction data collection. G. M. Sheldrick is thanked for valuable discussions and for providing the SHELXE program. This work was funded by projects MIT-Pt/BS-BB/1014/2008 from the MIT-Portugal Program and PTDC/EBB-BIO/098352/2008 from FCT, Portugal. IHS is the recipient of a $\mathrm{PhD}$ grant from FCT (SFRH/BD/36582/2007). DKN is an HHMI Investigator.

\section{References}

Croal, L. R., Jiao, Y. \& Newman, D. K. (2007). J. Bacteriol. 189, 1774-1782.

DeLano, W. L. (2002). PyMOL. http://www.pymol.org.

Emsley, P., Lohkamp, B., Scott, W. G. \& Cowtan, K. (2010). Acta Cryst. D66, 486-501.

Kabsch, W. (2010). Acta Cryst. D66, 125-132.

Kantardjieff, K. A. \& Rupp, B. (2003). Protein Sci. 12, 1865-1871.

Kappler, A., Pasquero, C., Konhauser, K. O. \& Newman, D. K. (2005). Geology, 33, 865-868.

Matthews, B. W. (1968). J. Mol. Biol. 33, 491-497.

Pape, T. \& Schneider, T. R. (2004). J. Appl. Cryst. 37, 843-844.

Saraiva, I. H., Newman, D. K. \& Louro, R. O. (2012). J. Biol. Chem. 287, 25541-25548

Sheldrick, G. M. (2008). Acta Cryst. A64, 112-122.

Sheldrick, G. M. (2010). Acta Cryst. D66, 479-485.

Weiss, M. S. (2001). J. Appl. Cryst. 34, 130-135.

Widdel, F., Schnell, S., Heising, S., Ehrenreich, A., Assmus, B. \& Schink, B. (1993). Nature (London), 362, 834-836.

Xiong, J., Fischer, W. M., Inoue, K., Nakahara, M. \& Bauer, C. E. (2000). Science, 289, 1724-1730. 